\title{
Intervenção da Terapia Ocupacional na Síndrome de Cri-du-Chat: estudo de caso
}

\author{
Gabriela Caseiro $^{\mathrm{a}}$, Flavia Manho ${ }^{\mathrm{a}}$, Daniela Baleroni Rodrigues Silva ${ }^{\mathrm{b}}$, Luzia Iara Pfeifer $^{\mathrm{c}}$ \\ aTerapeuta ocupacional, Faculdade de Medicina de Ribeirão Preto - FMRP, Universidade de São Paulo - USP, \\ Ribeirão Preto, SP, Brasil \\ ${ }^{\text {b}}$ Terapeuta ocupacional, Supervisora de Estágio em Infância e Adolescência do curso de graduação em Terapia \\ Ocupacional, Faculdade de Medicina de Ribeirão Preto - FMRP, Universidade de São Paulo - USP, \\ Ribeirão Preto, SP, Brasil \\ 'Docente do curso de Terapia ocupacional, dos Progaramas de Pós-graduação em Neurologia da FMRP-USP e \\ de Saúde Pública da Escola de Enfermagem, Faculdade de Medicina de Ribeirão Preto - FMRP, Universidade \\ de São Paulo - USP, Ribeirão Preto, SP, Brasil. Bolsista Produtividade CNPq Nível 2
}

\begin{abstract}
Resumo: A Síndrome de Cri-du-Chat é uma anomalia cromossômica que pode resultar em diversos acometimentos, incluindo atraso no desenvolvimento neuropsicomotor e deficiência intelectual. Este estudo de caso descreve a intervenção terapêutica-ocupacional com uma criança com Síndrome de Cri-du-Chat que foi acompanhada em atendimentos semanais, em um hospital escola do interior paulista entre 2 e 4 anos de idade. Para descrição do caso foram utilizados os registros no prontuário, relatos de familiares, bem como os dados dos atendimentos de Terapia Ocupacional. Na avaliação inicial identificou-se que a criança era hipotônica, ficava em pé com apoio e explorava objetos levando-os à boca. Os objetivos terapêutico-ocupacionais pautaram-se na estimulação das habilidades de desempenho sensório-motoras (processamento perceptual, neuro-músculo-esquelético e motor) e de integração cognitiva e componentes cognitivos (espectro de atenção, sequenciamento, aprendizado), por meio da estimulação do faz de conta e participação nas atividades de vida diária. Além de tais aspectos, foi necessário intervir diretamente no contexto escolar e familiar da criança, orientando os pais a evitar a superproteção. Por meio do trabalho conjunto entre equipe multiprofissional e familiares foi possível contribuir para a melhora das funções do corpo, possibilitando ampliação das atividades e participação, considerando os fatores pessoais e ambientais da criança, com consequente alta dos atendimentos ambulatoriais de Terapia Ocupacional.
\end{abstract}

Palavras-chave: Síndrome de Cri-du-Chat, Terapia Ocupacional, Desempenho Ocupacional.

\section{Occupational Therapy Intervention in a child with Cri-du-Chat Syndrome: a case study}

\begin{abstract}
Cri-du-Chat syndrome is a chromosomal abnormality that can result in several damages including developmental delay and intellectual disability of the affected child. This case study describes the occupational therapy intervention in a child with Cri-du-chat syndrome that was followed from two to four years old, in weekly sessions, at a school hospital in the state of São Paulo. Data from medical records, family reports, and occupational therapy sessions were used for case description. The initial assessment showed that the child was hypotonic, stood up only with support, and explored objects by taking them to the mouth. Occupational Therapy assistance aimed to stimulate sensorimotor performance skills (perceptual, neuromuscleskeletal and motor processing) and cognitive integration and components (attention spectrum, sequencing, and learning), through make-believe activities and participation in the activities of daily living (ADL). Moreover, it was also necessary to intervene directly in the school context and the child's family, advising parents to avoid overprotection. Through the joint
\end{abstract}

Autor para correspondência: Luzia Iara Pfeifer, Departamento de Neurologia, Psiquiatria e Psicologia Médica, Faculdade de Medicina de Ribeirão Preto, Universidade de São Paulo, Av. Bandeirantes, 3900, Monte Alegre, CEP 14049-900, Ribeirão Preto, SP,

e-mail: luziara@fmrp.usp.br

Recebido em 22/2/2010; $1^{\text {a }}$ Revisão em 24/2/2011; $2^{\text {a }}$ Revisão em 7/7/2011; Aceito em 19/9/2011. 
effort of the multidisciplinary team and the child's family, it was possible to contribute to the improvement of bodily functions, allowing an increase in activities and participation, considering the child's personal factors and environmental conditions, with consequent discharge from ambulatory attendance of occupational therapy.

Keywords: Cri-du-Chat Syndrome, Occupational Therapy, Occupational Performance.

\section{Introdução}

A Síndrome de Cri-du-Chat foi descrita em 1963 pelo cientista francês Dr. Jérôme Lejeune (MACHADO et al., 2007). É uma anomalia cromossômica causada pela perda do braço curto do cromossomo $5 \mathrm{p}$ e, portanto, é também denominada de síndrome do cromossomo $5 \mathrm{p}-$ (menos) (MAINARDI et al., 2006). A perda de material genético pode ser parcial, apenas um pequeno fragmento, ou total, com perda de todo braço curto do cromossomo 5 (WIEDEMANN, 1997). A anomalia afeta cerca de 1 em 50 mil nascidos no mundo e $1 \%$ dos indivíduos com deficiência intelectual (MAINARDI et al., 2006).

Uma pequena minoria dos casos de Síndrome de Cri-du-Chat corresponde à herança genética dos pais, teria origem secundária a uma segregaçáo desigual de uma translocação parental, a qual decorre de alguma falha durante a divisão celular, sem motivo aparente. Assim, como não houve perda do material genético, um dos pais apresenta translocaçáo equilibrada do cromossomo, porém não possui a síndrome e, provavelmente, só descobrirá ser portador da síndrome ao ter um filho afetado (BURNS; BOTTINO, 1991). Oitenta e cinco por cento dos casos da síndrome são resultado de novas deleçóes esporádicas (VASCONCELOS et al., 2007).

O choro das crianças com a síndrome é semelhante ao choro agudo de um gato, o que explica ser conhecida também como síndrome do miado de gato (BERTOLA; ALBANO; KIM, 2004). Esse choro é decorrente de má formaçáo da laringe e se torna menos característico conforme o crescimento da criança, dificultando, assim, o diagnóstico por meio desse sinal clínico em pacientes de maior idade (MUNIZ; BASTOS; AMARAL, 2002). Pode-se ainda acrescentar deficiência intelectual e atraso no desenvolvimento neuropsicomotor severo como características constantes em todos os casos (MUNIZ; BASTOS; AMARAL, 2002). As crianças começam a andar tardiamente, normalmente após os 3 anos, e geralmente esse andar é desajeitado e aparentemente inábil (MAINARDI et al., 2000).

As limitaçôes no desenvolvimento cognitivo podem variar de moderadas a severas (FALKENBACH; DREXSLER; WERLE, 2006). Muitas crianças com a síndrome podem desenvolver habilidades sociais normalmente vistas em crianças de 5 a 6 anos de idade e grau de independência moderado nas tarefas de autocuidado (WILKINS; BROWN; WOLF, 1980). Em estudo realizado por Cornish et al. (1999), ao examinarem 21 crianças de 5 a 15 anos com o Wechsler Intelligence Scale for Children - III (WISC - III), identificaram que todas apresentaram quociente de inteligência (QI) menor que 50. Porém há relatos de casos de outras crianças com a síndrome com quocientes de inteligência igual a 66 (SMITH et al., 1990).

Há estudos que tentaram correlacionar o desenvolvimento dessas crianças nos aspectos sociais, comportamentais e psicomotores com as condiçôes genéticas da síndrome $5 \mathrm{p}-\mathrm{S}$ (variando em caso de deleção e translocação), porém os dados são controversos (MARINESCU et al., 1999; MAINARDI et al., 2006). Marinescu et al. (1999) não encontraram correlação entre o tamanho da deleçáo e o nível de desenvolvimento em 50 participantes com a síndrome, enquanto Mainardi et al. (2001) relatou aumento da severidade do retardo psicomotor e de outras manifestaçóes clínicas em relação ao tamanho da deleção em 62 pacientes com deleçóes terminais. Outros autores relatam que crianças com a translocação do cromossomo $5 \mathrm{p}$ apresentavam comportamento social mais prejudicado quando comparadas com aquelas com deleção de tal cromossomo (WILKINS et al., 1983; DYKENS; CLARKE, 1997).

É comum essas crianças apresentarem problemas de comportamento, hiperatividade, balançarem muito a cabeça, morderem e beslicarem pessoas ao seu lado, assim como desenvolver obsessão por determinados objetos, sendo comum o fascínio por puxar cabelos (CORNISH; PIGRAM, 1996).

Observa-se, portanto, que a síndrome de Cri-duChat pode comprometer funções do corpo (funçôes fisiológicas dos sistemas corporais) e estruturas do corpo (partes anatômicas do corpo). As funções mentais, como atenção, são mais acometidas em decorrência da síndrome (DYKENS; HODAPP; FINUCANE, 2000; DYKENS; CLARKE, 1997), assim como as funçóes neuromusculoesqueléticas e as relacionadas ao movimento, levando ao atraso neuropsicomotor (MAINARDI et al., 2001).

Porém, é importante destacar que a incapacidade ${ }^{1}$ de uma pessoa é caracterizada como uma interação dinâmica entre os estados de saúde (doenças, lesôes, traumas) e os fatores contextuais que representam 
as circunstâncias nas quais o indivíduo vive (fatores ambientais e pessoais). Um ambiente com barreiras ou sem facilitadores vai restringir o desempenho do indivíduo, por outro lado, ambientes mais facilitadores podem melhorar esse desempenho (ORGANIZAÇÃO..., 2003).

Nesse sentido, a terapia ocupacional tem por objetivo favorecer o engajamento dos indivíduos em suas ocupaçôes e, para que o indivíduo possa desempenhá-las, é necessário considerar suas habilidades de desempenho, como aquelas relacionadas a aspectos sociais, de comunicação, cognitivos, assim como o contexto na qual a ocupação é desempenhada, as demandas da atividade, as funçóes e estruturas do corpo do cliente (AMERICAN..., 2008).

Destaca-se ainda a necessidade de atuação de uma equipe multiprofissional junto a crianças com a síndrome de Cri-du-Chat e, nesta pesquisa, destaca-se o papel do terapeuta ocupacional como favorecedor do desempenho de ocupaçóes pertinentes ao universo infantil, principalmente nas áreas relacionadas ao brincar, educação, lazer, participação social e atividades de vida diária.

\section{Objetivo}

Descrever o processo de intervenção terapêuticaocupacional com uma criança com a Síndrome de Cri-du-Chat realizada em hospital terciário do interior paulista.

\section{Metodologia}

Participante: B. P. F., sexo feminino, nasceu em 8 de abril de 2004, é a segunda filha de um casal não consanguíneo. Mora com os pais e irmão, dois anos mais velho, em uma chácara em uma cidade de porte médio no interior do estado de Sáo Paulo. Seus avós maternos moram em uma casa nos fundos do mesmo terreno.

Não houve intercorrências na gestação, a criança nasceu de parto normal com 36 semanas, $2,2 \mathrm{~kg}$ e apgar 2/7/9. Além disso, apresentou hipoglicemia ao nascer e dificuldade respiratória com 15 dias de vida, porém a ressonância magnética não identificou qualquer tipo de alteração.

No exame clínico após o nascimento, apresentava as seguintes características: braquicefalia, face atípica, olhos azuis com epicanto inferior, ausência de fixação do olhar, nariz com dorso curto, orelha de implantação baixa com lobos salientes, micrognatia, pescoço curto, tórax com escudo, prega palmar única e hipotonia muscular principalmente distal. Seu choro era fraco e se assemelhava ao de um gato, principalmente até os primeiros 6 meses de vida. B. P. F. apresentou atraso no desenvolvimento motor, sendo que aos 4 meses, como náo sustentava a cabeça, iniciou atendimento fisioterápico em um hospital público terciário na cidade natal. Ela sustentou a cabeça somente aos 6 meses, sentou sem apoio aos 11 meses, ficou em pé com apoio e passou a verbalizar algumas sílabas com 1 ano e 2 meses. Quando a criança tinha aproximadamente um ano foi confirmado o diagnóstico da Síndrome de Cri-du-Chat, pela deleçáo de parte do cromossomo $5 \mathrm{p}$, e feito encaminhamento pelo serviço de genética médica para atendimento no Ambulatório de Terapia Ocupacional do hospital citado. A criança foi avaliada em julho de 2005, mas aguardou em lista de espera, iniciando atendimentos semanais no referido ambulatório somente em março de 2006, época em que estava com 1 ano e 11 meses.

\section{Procedimento de coleta e análise de dados}

As informaçōes sobre o histórico clínico da criança foram coletadas a partir do prontuário médico, do relato da mãe e da avó, e aquelas relacionadas à intervenção da Terapia Ocupacional foram obtidas por meio do prontuário. A criança foi atendida por uma terapeuta ocupacional contratada pelo referido hospital de março de 2006 a dezembro de 2007 e, no período de fevereiro a dezembro de 2008 , a intervenção foi realizada por duas acadêmicas do $6^{\circ}$ semestre do curso de Terapia Ocupacional participantes desta pesquisa, sob supervisão de uma terapeuta ocupacional e uma docente, como parte das disciplinas Terapia Ocupacional Aplicada à Infância e Adolescência e Terapia Ocupacional Aplicada às Condiçôes Clínicas da Infância e Adolescência.

As intervençôes terapêuticas-ocupacionais foram registradas no prontuário clínico da criança após cada atendimento, sendo feitas reavaliaçóes a cada 6 meses. Quando se identificavam os ganhos obtidos, novos objetivos terapêuticos eram elaborados e delimitadas as estratégias para tais metas serem alcançadas. Foram utilizados formulários de avaliaçáo do próprio serviço de Terapia Ocupacional do hospital, que aborda como pontos relevantes a serem observados: as áreas de ocupação relacionadas ao universo infantil (brincar, educação, atividades de vida diária, lazer e participação social), bem como as habilidades de desempenho que podem interferir no desempenho de tais ocupaçôes. As habilidades de desempenho avaliadas são as: práxicas e motoras, percepto-sensoriais, de regulação emocional, cognitivas, sociais e de comunicação. Por meio da utilização de tal formulário de avaliação foi possível realizar comparaçóes com avaliaçóes anteriores e observar os ganhos alcançados para, dessa forma, formular estratégias para potencializar o engajamento nas ocupaçôes infantis. 
O presente relato seguiu as normas éticas de pesquisas com seres humanos e, portanto, a divulgação desse caso clínico foi aprovada pelo Comitê de Ética do referido hospital.

\section{Resultados}

\subsection{Evolução clínica da criança dos 2 aos 4 anos de idade}

Desde o nascimento, a criança foi acompanhada pelo serviço de neurologia e pediatria e, a partir dos 2 anos, também passou a fazer atendimento fonoaudiológico, com objetivo de estimular a linguagem oral, sistema orofacial e funçóes de mastigação e deglutição. Fez atendimento fisioterápico semanalmente, do segundo semestre de 2004 até dezembro de 2005, quando adquiriu marcha independente, ainda que com base alargada.

$\mathrm{Na}$ avaliação inicial realizada pelo serviço de Terapia Ocupacional em 20 de julho de 2005, quando a criança tinha 1 ano e 3 meses, identificou-se que ela não engatinhava e não ficava em pé sem apoio em função da hipotonia. Demonstrava iniciativa nas brincadeiras realizadas, imitava gestos, porém só explorava os objetos levando-os à boca.

A intervençáo inicial da Terapia Ocupacional, a partir de março de 2006, se deu por meio da estimulação da área de ocupação do brincar e das habilidades de desempenho percepto-sensoriais (processamento sensorial tátil e perceptual, esquema corporal, discriminaçãao direita-esquerda, posiçáo no espaço), dos aspectos motores (coordenação motora fina e grosseira, integração bilateral, integração viso-motora, resistência) e da integração cognitiva e das habilidades cognitivas (espectro de atençáo, sequenciamento, aprendizado). Tais habilidades foram estimuladas através da realização de atividades de vida diária apropriadas para a idade (tirar sapatos de forma independente, realizar a alimentação fazendo uso de colher), da participação em brincadeiras como gincanas e estimulação do faz de conta (dar comida, banho na boneca).

Após três meses de intervenção, a criança ainda apresentava dificuldades para manter-se concentrada nas atividades no tempo esperado para sua faixa etária e era bastante agitada. Aos 2 anos e 6 meses, ela passou a controlar os esfíncteres e apresentar maior atenção e interesse por objetos como boneca e telefone de brinquedo. Aos 2 anos e 7 meses (novembro de 2006), passou a frequentar escola de educação infantil durante meio período, mantendo-se os atendimentos de Terapia Ocupacional e Fonoaudiologia. Aos 3 anos, a criança começou a verbalizar algumas palavras e a mostrar-se mais atenta e concentrada nas atividades, a reconhecer formas e a seguir as etapas de atividades. Em função da dificuldade na marcha e do desequilíbrio, passou a utilizar bota ortopédica por indicação de um ortopedista no período de 2007 a 2008.

\subsection{Descrição dos atendimentos terapêuticos ocupacionais da criança aos 4 anos de idade}

Em março de 2008 foi realizada a reavaliação do caso pelas estagiárias de Terapia Ocupacional e pela supervisora, a fim de identificar novos objetivos terapêuticos e traçar as estratégias de intervenção. Foi possível identificar que na área de ocupaçáo do brincar o que mais interessava a criança era a boneca (alimentava e dava banho), que ela identificava figuras de objetos e situaçóes de seu cotidiano, discriminava frequentemente cores e formas, apesar de não nomeá-las. Em relação às habilidades de desempenho motoras, apreendia e soltava ativamente objetos, apresentava função bimanual e coordenação viso-motora compatíveis com o esperado para a faixa etária, realizava todas as trocas posturais com independência, sentava sem apoio, andava com base alargada e subia em móveis com auxílio.

A criança apresentava hipotonia generalizada, com maior acometimento nos membros inferiores, desequilíbrio, limitaçóes na coordenação motora grossa (pular, correr, dançar, arremessar), tendência à preensão palmar, pronação do antebraço e dificuldades na fala. Apesar dos registros em prontuário apontarem evolução na atenção e concentração, ainda notavam-se déficits nesses aspectos, assim como no julgamento e no raciocínio. Em relação à área de ocupação escolar, foi relatado pela professora que a criança apresentava dificuldades na realização de atividades pedagógicas compatíveis com sua faixa etária. Em relação ao processamento perceptual, apresentava atraso na orientação temporo-espacial e no esquema corporal.

Nas atividades de vida diária (AVDs), a criança era capaz de colocar e retirar o sapato com auxílio verbal, vestir e despir as roupas, alimentar-se de forma independente fazendo uso de garfo ou colher sem derramar, embora fosse relatado pela máe que ela desempenhava tais tarefas sozinha somente nos finais de semana.

$\mathrm{Na}$ área de ocupação de lazer e participação social foi constatado que a criança participava de eventos e outras situações sociais, mas com limitações nas relações sociais em função de comportamentos inadequados como puxar cabelos, além de déficits na comunicação verbal e não verbal. Dessa forma, os pais estavam sempre supervisionando a criança em tais situações, com tendência a superprotegê-la.

Foi realizada uma visita escolar com a participação das estagiárias de Terapia Ocupacional, da 
fonoaudióloga e da professora que acompanhava a criança, com objetivo de delimitar estratégias comuns para favorecimento da independência e desenvolvimento da criança. Durante a visita à escola, foi possível perceber que a criança participava de todas as atividades oferecidas pela professora, porém as realizava com dificuldade, sendo necessário auxílio constante da professora assistente. A classe de B. P. F. era voltada para a faixa etária de 2 a 4 anos e ela, apesar de ser a criança mais velha da turma, brincava preferencialmente com as crianças de 2 anos. Foi identificado que sua carteira localizava-se de frente para as outras crianças na sala de aula, o que dificultava que mantivesse a atençáo nas atividades realizadas. Dessa forma, foi sugerido que sua carteira fosse colocada de frente para a lousa, minimizando estímulos visuais e auditivos que pudessem interferir na sua atenção e concentração em sala de aula. Foi relatado pela professora que o irmáo da criança, que frequentava a mesma escola, assumia o papel de cuidador dela e que isso interferia nas relaçóes dela com os colegas da escola.

A partir das informaçóes obtidas no processo de reavaliação, anamnese e visita à escola (março de 2008), definiram-se como objetivos terapêuticos ocupacionais a melhoria no desempenho nas áreas de ocupação: do brincar, da educação, da participação social e das atividades de vida diária. Para alcançar tais objetivos, foram estimuladas as habilidades de desempenho motoras (coordenação motora fina e global, integração bilateral e equilíbrio), habilidades neuro-músculo-esqueléticas (propriocepção, tônus dos membros superiores e reação de proteçáo), cognitivos (atenção, concentração, raciocínio), processamento perceptual (orientação espacial e esquema corporal) e habilidades psicossociais (cooperação e habilidades interpessoais).

As estratégias utilizadas englobaram jogos que incluíam conceitos de formas, cores, animais e frutas, atividades com o uso de diferentes texturas, como colagem de grãos, pintura com as mãos, utilização de diferentes papéis, massa de modelar, brincadeiras de faz de conta, músicas com gestos para, dessa forma, estimular habilidades de desempenho deficitárias citadas anteriormente.

Durante reavaliação feita com a criança após seis meses de atendimento (setembro de 2008), foi possível identificar progresso em relaçáo aos aspectos cognitivos (maior atenção e concentração, julgamento, sequenciamento e raciocínio), nas habilidades de desempenho motoras (coordenaçáo motora grossa, fina e menor desequilíbrio) e em suas habilidades psicossociais, permitindo maior independência nas áreas de desempenho ocupacional do brincar, AVDs, educação e participação social. A criança passou a utilizar com maior frequência o garfo e, em certos momentos, a faca sem ponta, passou a retirar e colocar blusas, calças com elástico na cintura, sapatos e sandálias e a manipular de forma satisfatória velcros, zíperes e botôes. Também passou a utilizar o vaso sanitário de forma independente.

$\mathrm{Na}$ escola, passou a se relacionar com crianças de idade mais próxima à sua e a realizar as atividades propostas pela professora junto com os colegas de classe, não permanecendo mais em outras atividades paralelas e realizando de forma participativa o conteúdo exigido pela escola. Nos jogos e no faz de conta apresentou aumento de atenção, concentração, raciocínio e sequenciamento. Quanto aos aspectos motores, foi observado aumento do equilíbrio e da reação de proteção, com diminuição da base de apoio em sua marcha.

Em função dos ganhos obtidos nos componentes cognitivos (atenção, concentração, sequenciamento), a partir de setembro de 2008, o foco de intervenção passou a ser o treino de atividades de vida diária (AVDs) pela criança, com a participação do cuidador, para realização de orientaçôes, pois evidenciavam-se superproteção da criança e pouco estímulo à independência. Para o treino de AVDs foram usados jogos simbólicos (principalmente com boneca), circuitos, desfile de modas, preparação de lanche e festa de aniversário, além do treino das atividades propriamente ditas, tais como utilizar o banheiro, escovar os dentes, alimentar-se e vestir-se.

Alguns atendimentos foram realizados com a presença do irmão, a fim de fortalecer a relação e o papel de "brincante" entre eles e visando inibir o papel de cuidador que o mesmo exercia em relação à irmã. Sempre que possível foram realizadas atividades que envolvessem cooperação, relações interpessoais e temas relacionados a datas comemorativas como dia do índio, dia das máes e festa junina.

Foi possível identificar que o trabalho feito com os cuidadores da criança contribuiu para os resultados positivos, pois gradualmente eles passaram a permitir que a criança desempenhasse suas atividades rotineiras de forma mais independente. Em dezembro de 2008, a criança recebeu alta dos atendimentos semanais da Terapia Ocupacional e passou a ser atendida em retornos semestrais, para acompanhamento do desenvolvimento e identificaçáo da necessidade de orientaçóes quanto ao seu desempenho ocupacional. No momento de elaboração deste relato, a criança estudava em escola regular e mantinha os atendimentos fonoaudiológicos

\section{Discussão}

Segundo Muniz, Bastos e Amaral (2002) e Sarimski (2003), apesar da limitação devido à deficiência intelectual e retardo neuropsicomotor 
que a criança com Síndrome de Cri-du-Chat pode apresentar, a estimulação precoce possibilita a ela melhor desenvolvimento, adaptação e aceitaçáo da sociedade. Assim, pôde-se perceber que a Terapia Ocupacional teve importante papel nesse processo, associada ao trabalho em equipe (Fonoaudiologia, Fisioterapia, Pedagogia), possibilitando que a criança evoluísse na coordenaçáo motora grossa e fina, nas habilidades cognitivas e nas perceptivas, nas atividades de vida diária e nas relaçóes interpessoais.

Em crianças com deficiências, o enfoque terapêutico deve ser sobre a função, para que se possam reduzir as limitações funcionais e melhorar o bem-estar (LOLLAR; SIMEONSSON, 2002). A partir da abordagem biopsicossocial proposta pela Classificação Internacional da Funcionalidade, Incapacidade e Saúde - CIF (ORGANIZAÇÃO..., 2003), a incapacidade não é vista somente como resultado de uma condição de saúde, mas como consequência da influência e dos efeitos de fatores sociais, psicológicos e ambientais (SAMPAIO; LUZ, 2009).

Por essa razão, a intervenção junto à família foi de fundamental importância para o desenvolvimento da criança, especialmente em relação à melhora no desempenho das atividades de vida diária que antes eram desempenhadas pelos familiares. Segundo a CIF (ORGANIZAÇÃO..., 2003), aspectos facilitadores ou barreiras para a realizaçáo de atividades podem estar presentes nos contextos vivenciados pelas crianças. No caso de B. P. F, a superproteção dos pais se apresentou como uma barreira para a realização de suas atividades, porém, quando eles compreenderam a importância da autonomia da criança, bem como a necessidade de estabelecer limites, passaram a ser importantes facilitadores.

$\mathrm{Na}$ escola, o contato com diversas atividades e a vivência de relaçóes interpessoais com os colegas constituíram-se em facilitadores para o desenvolvimento de B. P. F, enquanto que barreiras como a superproteção do irmáo e o fato de estudar com crianças mais novas passaram a ser objetivos de intervenção no processo terapêutico.

Em relação ao brincar, que é entendido como uma importante área de desempenho ocupacional (AMERICAN..., 2008) e consiste em uma ocupação infantil significativa e fundamental (PARHAM; PRIMEAU, 2000), observa-se que a criança apresentou evoluçóes significativas comparando-se a avaliaçáo inicial e a final, no término dos atendimentos de Terapia Ocupacional. A literatura aponta diferenças significativas no brincar de crianças que têm a síndrome, principalmente no que se refere ao jogo simbólico, assim como no nível mental (SARIMSKI, 2003), diferenças cuja causa ainda é desconhecida. Sarimski (2003) avaliou o brincar simbólico de 10 crianças com a síndrome, com idade variando entre 24 e 82 meses, através de testes padronizados. Verificou-se que as crianças apresentaram comportamentos não relacionados ao tema do jogo, o que pode ser visto como precursor de problemas adaptativos. Hiperatividade e distrabilidade também foram notadas, bem como brincar exploratório pobre (dificuldade para colocar e retirar objetos de recipientes e manipular objetos).

No caso da criança participante do presente estudo, o brincar exploratório apresentava-se adequado, na medida em que ela manipulava objetos e colocava e retirava objetos de recipientes quando solicitada. Aos 4 anos relatava fatos simples do cotidiano, descrevia os objetos (grande, pequeno, pesado), iniciava brincadeiras, esperava a vez em atividades, após direcionamento inicial, elaborava planos simples para uma atividade de brincar com a terapeuta. No entanto, conforme observado no estudo de Sarimski (2003), a criança apresentava-se distraída durante as brincadeiras, relatava fatos do cotidiano de maneira empobrecida, assim como seu faz de conta e iniciativa eram limitados, sendo necessária estimulação constante.

Porém, ainda que em relação aos componentes acima citados avanços tenham sido obtidos no brincar, que passou a apresentar-se de forma mais adequada para a idade dela, a criança ainda se distraía facilmente, o que é compatível com os dados relatados na literatura (DYKENS; HODAPP; FINUCANE, 2000; DYKENS; CLARKE, 1997). Porém tal fato não a impedia de desempenhar as principais ocupaçóes relacionadas à infância, principalmente brincar, atividades de vida diária, educação, lazer e participação social. Ressalta-se a importância do acompanhamento da criança, mesmo após a alta da Terapia Ocupacional, em periodicidade semestral, para verificação de novas demandas de intervenção, a fim de que, se necessárias, sejam feitas orientaçóes aos professores e pais e intervençôes junto à criança.

Além disso, faz-se necessário ampliar a divulgação de pesquisas na área, englobando intervençôes desde a infância até a vida adulta, para que a síndrome, que é rara, seja mais bem conhecida e tratada de acordo com as evidências científicas.

\section{Referências}

AMERICAN OCCUPATIONAL THERAPY ASSOCIATION - AOTA. Occupational therapy practice framework: Domain and process. American Journal of Occupational Therapy, New York, v. 63, n. 6, p. 625-683, Nov 2008.

BERTOLA, D. R.; ALBANO, L. M. J.; KIM, C. A. Síndromes Genéticas e Cardiopatias. Revista da Sociedade de Cardiologia do Estado de São Paulo, São Paulo, v. 14, n. 3, p. 418-428, 2004. 
BURNS, G. W.; BOTTINO, P. J. Genética. Rio de Janeiro: Guanabara Koogan, 1991.

CORNISH, K. et al. Cognitive functioning in children with typical Cri-du- Chat (Çfp-) syndrome. Developmental Medicine and Child Neurology, London, v. 41, n. 4, p. 263-266, 1999. http://dx.doi.org/10.1017/ S0012162299000559

CORNISH, K. M.; PIGRAM, J. Developmental and behavioural characteristics of cri du chat syndrome. Archives of Disease in Childhood, London, v. 75, n. 5, p. 448-450, Nov 1996. http://dx.doi.org/10.1136/ adc.75.5.448

DYKENS, E.; CLARKE, D. Correlates of maladaptive behaviour in individuals with $5 \mathrm{p}$ - (Cri-du-Chat) syn drome. Developmental Medicine and Child Neurology, London, v. 39, n. 11, p. 752-756, Nov 1997.

DYKENS, E.; HODAPP, R.; FINUCANE, B. Genetics and Mental Retardation Syndromes. Baltimore: Paul H. Brookes, 2000.

FALKENBACH, A. T. P.; DREXSLER, G.; WERLE, V. O jogo e a síndrome do Miado do Gato. Revista Digital, Buenos Aires, v. 11, n. 96, p. 1, 2006.

LOLLAR, D. J.; SIMEONSSON, R. J. Diagnosis to Function: Classification for Children and Youths. Journal of Development \& Behavioral Pediatrics, Hagerstown, v. 26, n. 4, p. 323-330, Aug 2002. http://dx.doi. org/10.1097/00004703-200508000-00012

MACHADO, N. C. S. S. et al. Principais características clínicas da síndrome Cri-Du-Chat: revisão de literatura. In: ENCONTRO LATINO AMERICANO DE INICIAÇÃO CIENTÍFICA, 11.; ENCONTRO LATINO AMERICANO DE PÓS GRADUAÇÃO, 7., 2007, São José dos Campos. Anais... São José dos Campos: Universidade do Vale do Paraíba, 2007. p. 1668-1671.

MAINARDI, P. C. et al. Psychomotor development in cri du chat syndrome. Clinical Genetics, Copenhagen, v. 57, n. 6, p. 459-461, June 2000. http://dx.doi. org/10.1034/j.1399-0004.2000.570612.x

MAINARDI, P. et al. Clinical and molecular characterisation of 80 patients with 5 p-deletion: genotype- phenotype correlation. Journal of Medical Genetics, London, v. 38, n. 3, p. 151-158, Mar 2001. http://dx.doi.org/10.1136/jmg.38.3.151

MAINARDI, P. C. et al. The natural history of Cri-duChat syndrome. A report from the Italian Register. European Journal of Medical Genetics, Amsterdam, v. 49, n. 5, p. 363-383, Sept/Out 2006.
MARINESCU, C. et al. No relationship between the size of the deletion and the level of developmental delay in Cri-du-Chat syndrome. American Journal of Medical Genetics, New York, v. 86, n. 1, p. 66-70, Sept 1999. http://dx.doi.org/10.1002/(SICI)10968628(19990903)86:1<66::AID-AJMG13>3.0.CO;2-N

MUNIZ, J. W. C.; BASTOS, C. C.; AMARAL, R. O. Intervenção Fisioterapêutica na Síndrome do Cri-du-Chat. Lato Sensu, Belém, v. 4, n. 6, p. 112-119, 2002.

ORGANIZAÇÃO MUNDIAL DA SAÚDE - OMS. CIF: Classificação Internacional de Funcionalidade, Incapacidade e Saúde. Tradução do Centro Colaborador da Organização Mundial da Saúde para a Família de Classificaçôes Internacionais. São Paulo: EdUSP, 2003.

PARHAM, L. D.; PRIMEAU, L. A. Recreação e terapia ocupacional. In: PARHAM, L. D.; FAZIO, L. S. A recreação na terapia Ocupacional pediátrica. 1. reimp. São Paulo: Santos, 2000. p. 2-21.

SAMPAIO, R. F.; LUZ, M. T. Funcionalidade e incapacidade humana: explorando o escopo da classificação internacional da Organização Mundial da Saúde. Cadernos de Saúde Pública, Rio de Janeiro, v. 25, n. 3, p. 475-483, mar. 2009. http://dx.doi.org/10.1590/ S0102-311X2009000300002

SARIMSKI, K. Early play behavior in children with 5 p- (Cri-du-Chat) syndrome. Journal of Intellectual Disability Research, Oxford, v. 47, n. 2, p. 113-120, Jan 2003. http:// dx.doi.org/10.1046/j.1365-2788.2003.00448.x

SMITH, A. et al. Two cases of Cri-du-Chat syndrome with mild phenotypic effect but with different size of 5 p-deletion. Journal of Pediatric Child Health, Melbourne, v. 26, n. 3, p. 152-154, June 1990. http:// dx.doi.org/10.1111/j.1440-1754.1990.tb02414.x

VASCONCELOS, B. et al. Anormalidades cromossômicas nos pacientes atendidos em serviço de genética. Pediatria, São Paulo, v. 29, n. 1, p. 26-32, 2007.

WIEDEMANN, H. R. Atlas de sindromes clinicas dismórficas. 3. ed. São Paulo: Manole, 1997.

WILKINS, L.; BROWN J.; WOLF, B. Psychomotor development in 95 home-reared children with Cri-du-Chat syndrome. Journal of Pediatrics, Saint Louis, v. 97, n. 3, p. 401-405, 1980.

WILKINS, L. et al. Clinical heterogeneity in 80 homereared children with Cri-du-Chat syndrome. Journal of Pediatrics, Saint Louis, v. 102, n. 4, p. 528-533, Apr. 1983.

\section{Contribuição dos Autores}

Gabriela Caseiro e Flavia Manho: Participaram da coleta e análise dos dados, assim como da elaboraçáo do artigo. Daniela Baleroni Rodrigues Silva: Participou da supervisão das intervençōes, da coleta e análise dos dados, assim como da elaboração do artigo. Luzia Iara Pfeifer: Participou da orientação do trabalho, da análise dos dados e da elaboração do artigo.

\section{Notas}

${ }^{1}$ A Classificação Internacional de Funcionalidade, Incapacidade e Saúde (ORGANIZAÇÃO..., 2003) define incapacidade como termo que abrange deficiências, limitação de atividades ou restrição na participação. 